\title{
Effect of annealing process of iron powder on magnetic properties and losses of motor cores
}

\author{
FUZHENG YIN, HAIBO YANG ${ }^{\dagger}$, JIANJUN TIAN, DE-AN PAN, JIAN WANG and \\ SHENGEN ZHANG* \\ School of Materials Science and Engineering, \\ ${ }^{\dagger}$ School of Mechanical Engineering, University of Science and Technology Beijing, Beijing 100083, PRC
}

MS received 12 September 2009; revised

\begin{abstract}
Iron powder magnetic cores are used as soft magnetic rotors, in micro special motors such as BS brake motors, refrigerator compressor motors and brushless servo motors. Heat treatment of iron powder played an important role in the magnetic properties and loss of the motor cores. After the annealing process, the cracks and the pores on the surface of the powder decreased which in turn decreased the micro-hardness. The Vickers-hardness of the powder decreased from 50-42, while the resistance of the cores increased by $87 \%$ after annealing at $400^{\circ} \mathrm{C}$ for $30 \mathrm{~min}$. The amplitude permeability and magnetic loss of the cores reached the maximum and minimum values, respectively. The magnetic loss of the cores was separated into hysteresis loss and eddy current loss by Stoppels Method which were decreased by the annealing process.
\end{abstract}

Keywords. Reduced iron powders; annealing temperature; magnetic properties; magnetic loss.

\section{Introduction}

In recent years, iron powder based soft magnets (IPSM) are being used in motors replacing the existing laminated materials (Magnussen et al; Bayramli et al 2005; Hamler et al 2006) for its excellent magnetic properties such as higher permeability, higher saturation magnetic induction, lower cost, and for simplicity in manufacturing (Sowter 1987). The performance of the iron powder based soft magnets were determined by the composition, particle size, morphology and microstructure of the iron powder. Compared with other iron powders, the reduced iron powders had an advantage of lowest cost, which in turn greatly reduced the cost of the motor cores. However, the magnetic properties of the cores were lower than those of the pure iron powder based cores. Annealing process was usually required to minimize the deleterious effects on the magnetic performance of the core materials (Shokrollahi and Janghorban 2007). The annealing process of the iron powder as a pretreatment process improved the magnetic properties and lowered the loss of reduced iron powder based cores. The effect of the annealing temperature on the magnetic properties and losses of powder cores in alternating magnetic field is studied in this paper.

\footnotetext{
*Author for correspondence (zhangshengen@mater.ustb.edu.cn)
}

\section{Experimental}

Reduced iron powders were annealed at $300^{\circ} \mathrm{C}, 400^{\circ} \mathrm{C}$, $500^{\circ} \mathrm{C}, 600^{\circ} \mathrm{C}$ in argon atmosphere for $30 \mathrm{~min}$ and marked as samples ANN300, ANN400, ANN500, ANN600. The annealed powders and raw powders were compressed into ring cores of size $\mathrm{D} 27.06 \mathrm{~mm} \times \mathrm{d}$ $14.42 \mathrm{~mm} \times \mathrm{H} 10.06 \mathrm{~mm}$ in $500 \mathrm{MPa}$. The morphology and micro-hardness of iron powders were studied by scanning electron microscopy (SEM) and Vickershardness test instrument, respectively. Low-resistance measuring instrument was used to measure the resistivity of the cores. The amplitude permeability and the core losses were measured by high-frequency power test instrument from $20-100 \mathrm{kHz}$. Magnetic flux density $\mathrm{Bm}$ was $25 \mathrm{mT}$ with 20 core coils.

\section{Results and discussion}

Amplitude permeability $\left(\mu_{\mathrm{a}}\right)$ is an important performance index, which directly affects the application of the motor cores in the alternating magnetic field. Figures 1 and 2 show the effect of frequency and annealing temperature on the amplitude permeability of the cores. It can be seen that $\mu_{a}$ decreases with increase in frequency. After annealing process, $\mu_{\mathrm{a}}$ reaches a maximum value at $400^{\circ} \mathrm{C}$, and minimum value at $500^{\circ} \mathrm{C}$. The $\mu_{\mathrm{a}}$ of the cores of ANN300 and ANN600 are close to that of raw reduced iron powder based core. 
The $\mu_{\mathrm{a}}$ of the cores was improved by annealing at suitable temperatures because of the elimination of internal stresses and defects in the powders. The micro-hardness of the powder has direct correlation to the internal stresses and coercivity changes of powders, which explains the changes of $\mu_{\mathrm{a}}$ in the alternating magnetic field. Figure 3 shows the micro-hardness of the powders at different annealing temperatures. The Vickershardness of the sample ANN400 decreases from 50 to 42. This indicates the decrease of the internal stress and coercivity of the cores after annealing at $400^{\circ} \mathrm{C}$. But the Vickers-hardness of the sample ANN500 reaches the value 70, which increases the stress and coercivity of the powders. So the $\mu_{\mathrm{a}}$ of the ANN500 core is lower.

Figure 4 shows the microstructures of the iron powders. It can be seen in figure $4 a$ that there are many pores and defects on the surface of raw reduced iron powders; also there are many inner cracks, cavities and porosities

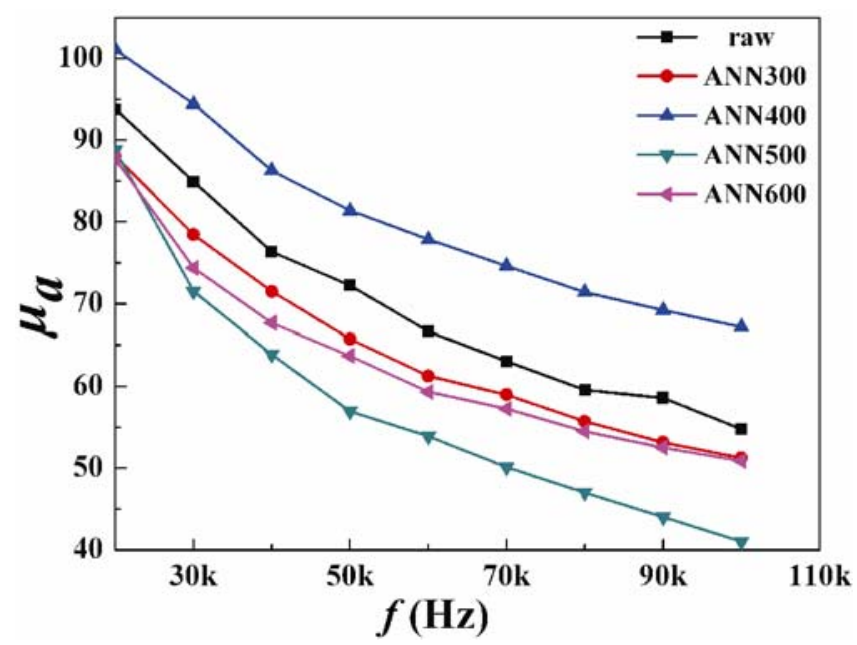

Figure 1. Effect of frequency on amplitude permeability.

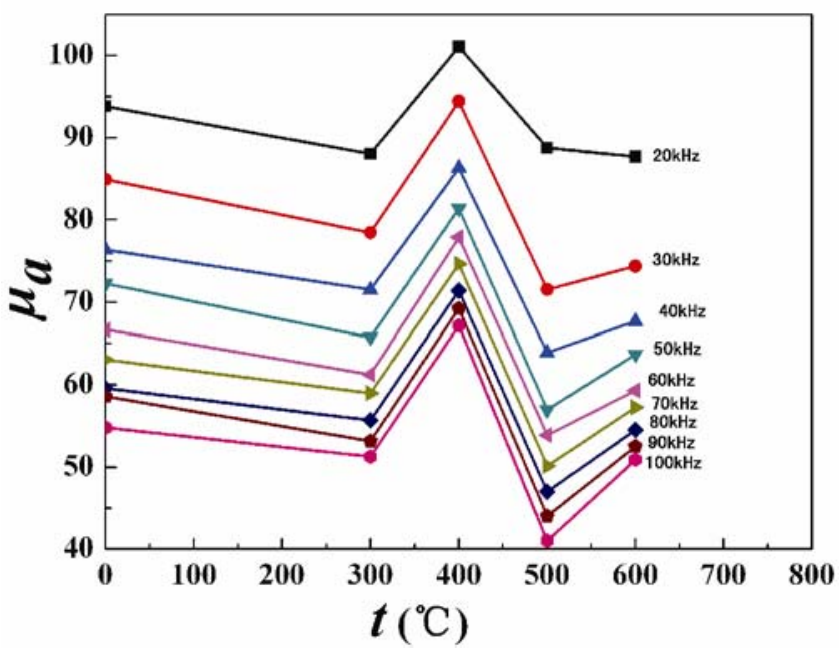

Figure 2. Effect of annealing temperature on amplitude permeability connected to the surface of particles. Every secondparticle is composed of many single-particles. As shown in figure $4 \mathrm{~b}$, the pores and defects on the surface of iron powders decrease after annealing at $400^{\circ} \mathrm{C}$. As the residual compacting stresses disappear in the progress, the contact resistance of the powder particles begins to increase, which causes increase in resistivity of the cores. As shown in figure $4 \mathrm{c}$, recrystallization of iron powders occurs when annealed at $500^{\circ} \mathrm{C}$. The contact areas have been increased; the sintering necks have formed and grown. Now the contact resistance of the powders has significantly decreased. Hence, the resistivity of the ANN500 core decreases.

Figure 5 shows the resistivity changes of the cores. It is seen that the resistivity of the ANN400 core is maximum, while that of the powders annealed at $500^{\circ} \mathrm{C}$ has dropped drastically.

Figures 6 and 7 show the magnetic loss $P \mathrm{cv}$ of the cores. $P \mathrm{cv}$ of the ANN400 core is the lowest while that of ANN500 core is the highest from $20-100 \mathrm{kHz}$. The $P \mathrm{cv}$ of ANN300 and ANN600 cores are close to that of raw reduced iron powder core.

Hysteresis loss $(P h)$ caused by the hysteresis effect in low-frequency and weak magnetic field can be written as:

$$
P h=\frac{4}{3} \times \mu_{0} \eta H m^{3} f,
$$

where $\eta$ is the Rayleigh constant. The formula shows the core loss caused by the hysteresis is proportional to the $f$ in alternating magnetic field and the cube of the amplitude magnetic field intensity $\mathrm{Hm}$.

The core loss caused by eddy current $(\mathrm{Pe})$ is (Hendricks et al 1991):

$$
P e=\frac{2}{3} \times \pi^{2} d^{2} f^{2} B m^{2} / \rho,
$$

where $B m$ is the amplitude of magnetic induction intensity, $\rho$ is conductivity and $d$ is the radius of eddy. The eddy loss is usually decreased by increasing the $\rho$ of the core.

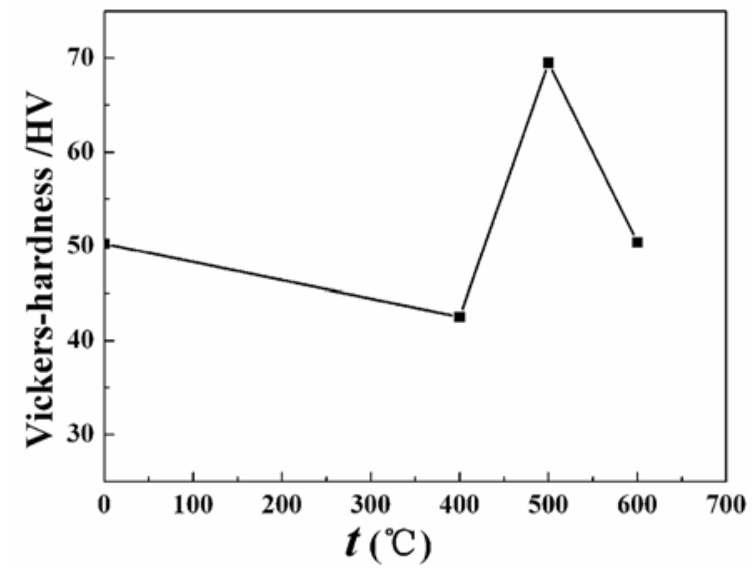

Figure 3. Vickers-hardness of the powders. 
The residual loss $P r$ is caused by magnetic after effect and resonances of the domain walls. The loss can be shown as under and is usually neglected in low-frequency and weak magnetic field.

$$
\operatorname{Pr}=C k\left(f B^{2}\right)^{a}
$$
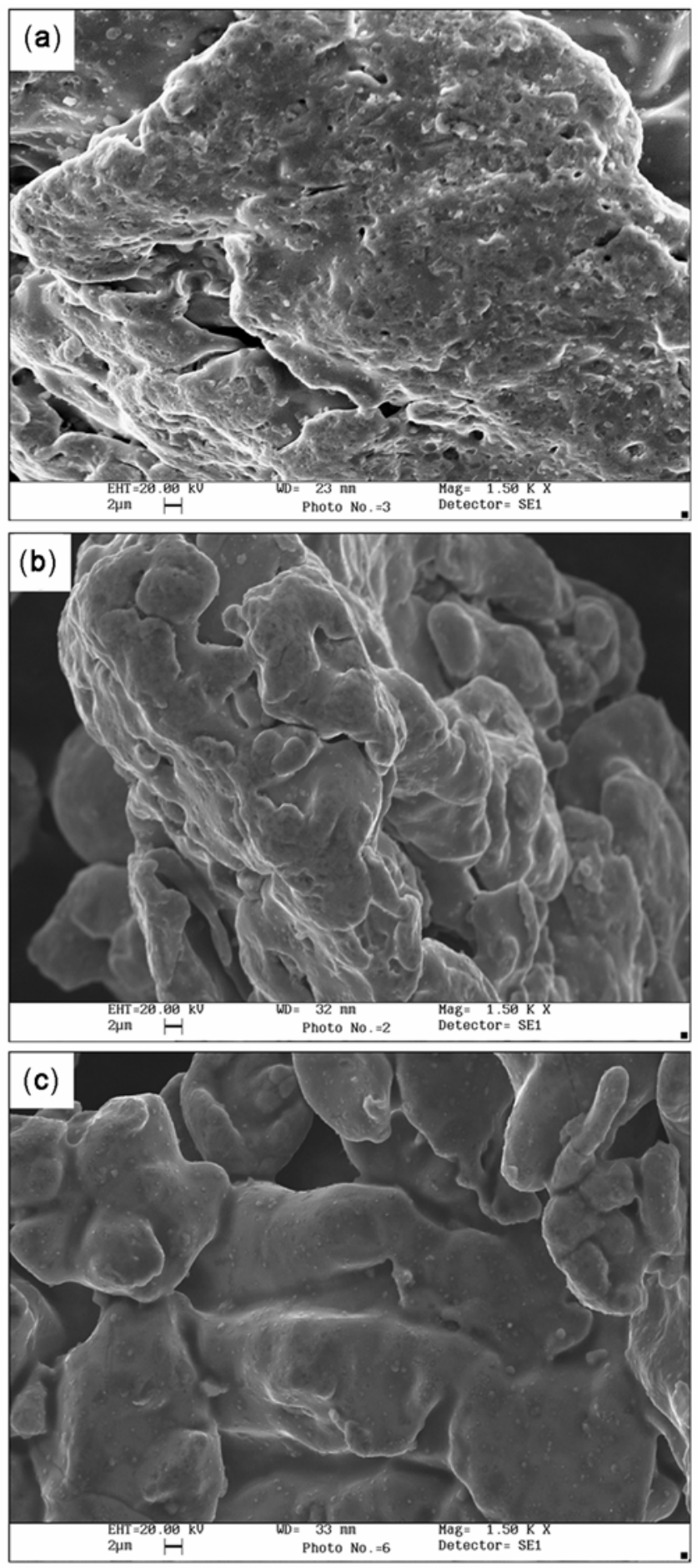

Figure 4. Micrograph of the powders (a) raw, (b) annealing at $400^{\circ} \mathrm{C}$ and (c) annealing at $500^{\circ} \mathrm{C}$.
The magnetic loss in low-frequency and weak alternating magnetic field can be described as (Legg 1936; Owens 1953):

$$
R m / \mu f L=2 \pi \tan \delta / \mu=e f+a B m+c,
$$

$R m$ is equivalent to the resistance of magnetic loss, $f$ is the frequency, $L$ is the inductance of magnetic components, $B m$ is the amplitude of the magnetic induction intensity and $\tan \delta$ is the quality factor, ef is the eddy loss where $e$ is the eddy loss coefficient, $a B m$ is the hysteresis loss where $a$ is the hysteresis loss coefficient and $c$ is the residual loss constant. In low frequency magnetic field, $c$ is independent of the frequency.

With the development of high permeability and low loss materials, a formula for magnetic loss has been put forward which is associated with the composition and structure of material:

$$
P t=P h+P e+P r=K h B^{2} f+K e B^{2} f^{2} d^{2} / \rho+P r,
$$

where $P t$ is the total core loss, $K h$ and $K e$ are coefficients. $\mathrm{Pr}$ can be ignored in low frequency magnetic field.

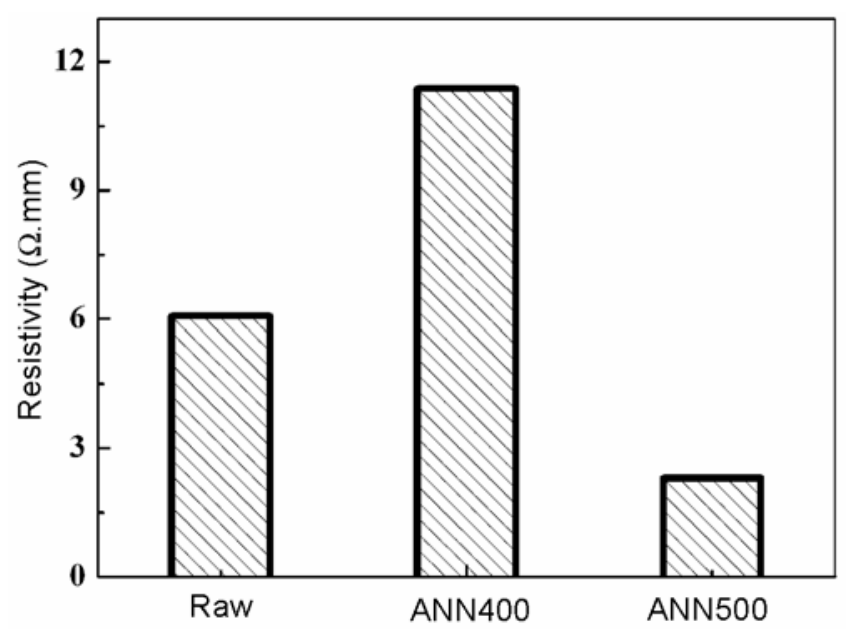

Figure 5. Resistivity of the cores.

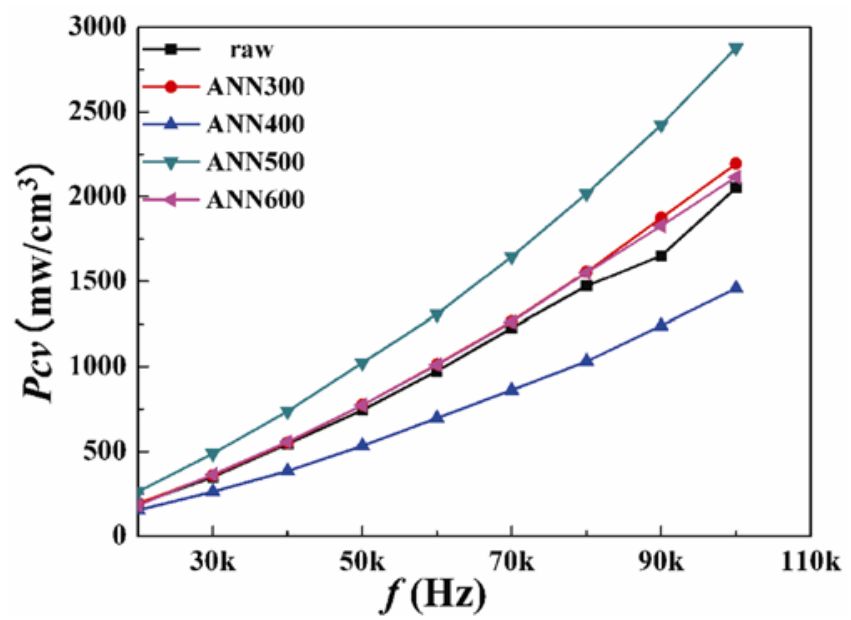

Figure 6. Magnetic loss of the cores. 


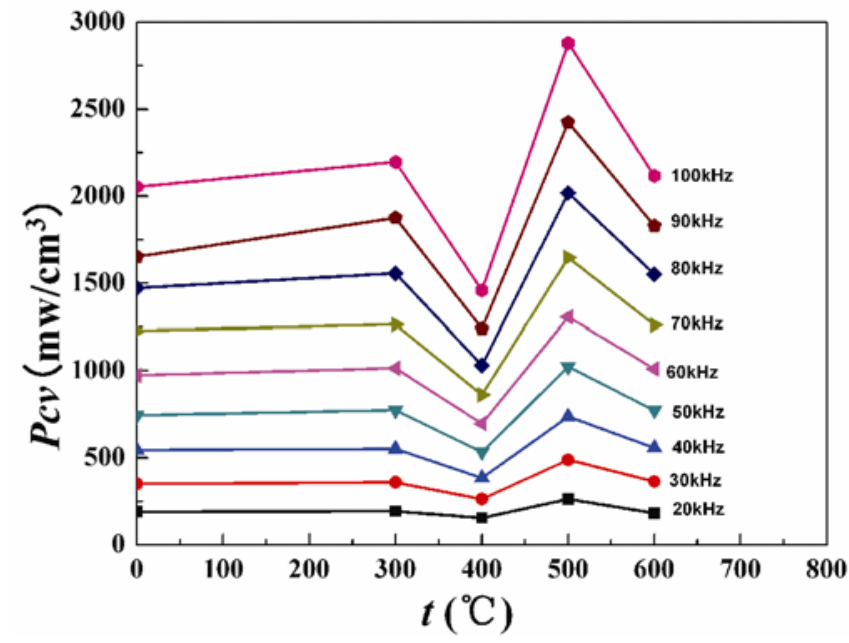

Figure 7. Effect of the annealing temperature on the magnetic loss of the cores.

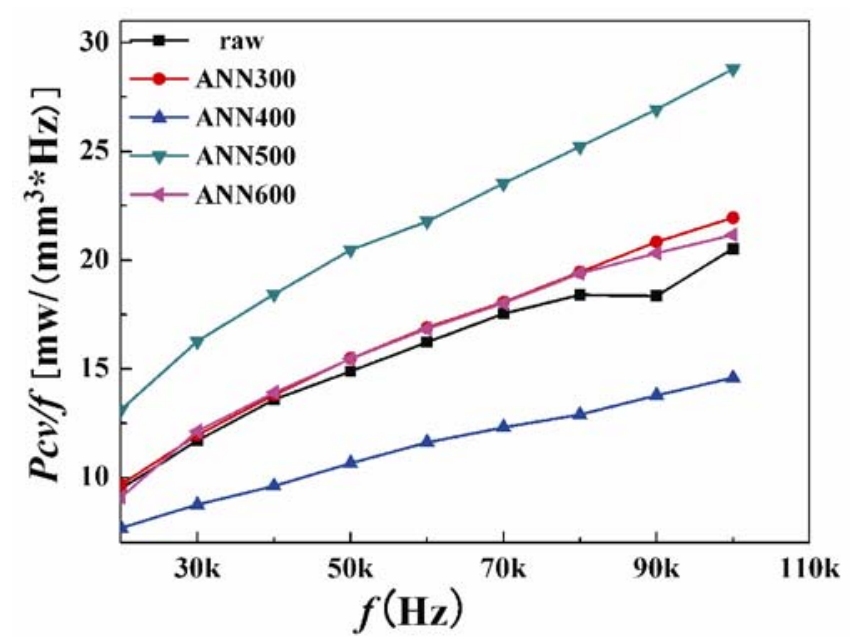

Figure 8. $\quad P c v / f-f$ curves from $20-100 \mathrm{kHz}$.

According to the Stoppels Loss-separating Methods (Stoppels 1996), an initial separation of the core loss can be made in certain magnetic flux density $B$ when $P t$ is plotted against $f$. Equation (5) can be rewritten as:

$$
P t \mid f=P h l f+P e l f=K h B^{2}+K e B^{2} f d^{2} / \rho .
$$

The way to separate core loss contributions is qualitatively demonstrated in figure 8, where the $P c v / f(P c v$ stands for the $P t$ in volume ratio) of powder cores is plotted against $f$. In the curve, $P h$ and $P e$ are given by the zero frequency intercept and the linear part respectively. The value of $P e$ is determined by the value of the linear slope. $P h / f$ is not completely frequency-independent, since the area of the hysteresis loop changes at higher frequencies. However, for not too high frequencies the approximation is reasonable (Stoppels 1996).
Figure 8 shows that the hysteresis loss $P h$ of ANN400 core is the lowest, and that of ANN500 core is highest. The $P h$ of ANN300 and ANN600 cores are very close to that of raw powder core. An effective way to lower the hysteresis loss $P h$ is to reduce the coercivity $H c$ of the material, which is similar to the mechanism to increase the amplitude permeability $\mu_{a}$. As shown in figures 3 and 4 , less pores, defects and low micro-hardness of iron powders annealed at $400^{\circ} \mathrm{C}$ have greatly decreased the coercivity $H c$, which significantly reduces the hysteresis loss of ANN400 powder core. The micro-hardness of the iron powders annealed at $500^{\circ} \mathrm{C}$ increases, hence the hysteresis loss $P h$ of ANN500 core also increases. The $P h$ of ANN300, ANN600 and raw reduced iron powder cores are close to each other because of a small change in their micro-hardness.

As shown in figure 8, the eddy loss $\mathrm{Pe}$ of ANN400 core is the lowest, and that of ANN500 core is the highest. The $P e$ of ANN300, ANN600 and raw powder cores are very close to each other because their linear slopes are similar. In (5), we see that the linear slopes of powder cores can be expressed as $K e B^{2} f d^{2} / \rho$. When the magnetic flux density $B$ is determined, the linear slope of the powder core is inversely proportional to $\rho$. As seen in figure 5 , the resistivity of ANN400 powder core sharply increases, which results in low linear slope and low eddy loss. On the contrary, the resistivity of ANN500 core is lower than others, and hence the linear slope and the eddy loss are higher.

\section{Conclusions}

After the reduced powders annealed at $400^{\circ} \mathrm{C}$, the amplitude permeability $\mu_{\mathrm{a}}$ of the core increases, while both the eddy loss and hysteresis loss of the core decrease. This is because when the micro-hardness and coercivity of powder decreases, the core resistivity increases. However, the amplitude permeability $\mu_{\mathrm{a}}$, the eddy loss and hysteresis loss of the powder core after the powder annealed at $500^{\circ} \mathrm{C}$ are contrary to those at $400^{\circ} \mathrm{C}$. According to the Stoppels Loss-separating methods, the core loss can be separated into hysteresis loss $(P h)$, the eddy current loss $(P e)$ and the residual loss $(P r)$. It is believed that both $P h$ and $P e$ of the cores decrease by the annealing process of the powder at $400^{\circ} \mathrm{C}$.

\section{Acknowledgements}

The authors gratefully acknowledge the support provided by National Natural Science Foundation of China (No. 50874010, No. 50802008), Key Projects in the National Science \& Technology Pillar Program of China (No. 2009BAE74B03). 


\section{References}

Bayramli E, Olgelioglu O and Ertan H B 2005 J. Mater. Process. Technol. 16183

Hamler A, Gorican V, Sustarsic B and Sirc A 2006 J. Magn. Magn. Mater. 304816

Hendricks C R, Amarakoon VWR and Sullivan D 1991 Ceram. Bull. 70817

Legg V E 1936 Bell Sys. Tech. J. 1539
Magnussen F, Svechkarenko D, Thelin P and Sadarangani $\mathrm{C}$ Analysis of a PM machine with soft magnetic composites core (Sweden: KTH-Royal Institute of Techno$\operatorname{logy})$

Owens C D 1953 Proc. IRE. 41359

Shokrollahi H and Janghorban K 2007 J. Mater. Process. Technol. 1891

Sowter G A V 1987 J. Audio Eng. Soc. 35760

Stoppels D 1996 J. Magn. Magn. Mater. 160323 